ROM2F-97/4

\title{
Surprises in Open-String Perturbation Theory
}

\author{
Augusto Sagnotti \\ Dipartimento di Fisica \\ Università di Roma "Tor Vergata" \\ I.N.F.N. - Sezione di Roma "Tor Vergata" \\ Via della Ricerca Scientifica, 100133 Roma ITALY
}

\begin{abstract}
The perturbative analysis of models of open and closed superstrings presents a number of surprises. For instance, variable numbers of antisymmetric tensors ensure their consistency via generalized GreenSchwarz cancellations and a novel type of singularity occurs in their moduli spaces. All these features are related, in one way or another, to the presence of boundaries on the world sheet or, equivalently, of extended objects (branes) interacting with the bulk theory in space time. String dualities have largely widened the interest in these models, that exhibit a wealth of generic non-perturbative features of String Theory.
\end{abstract}

Contribution to the Proceedings of the XXX Ahrenshoop Meeting, Buckow (Berlin), August 1996 


\title{
Surprises in Open-String Perturbation Theory
}

\author{
Augusto Sagnotti ${ }^{\text {** }}$ \\ aDipartimento di Fisica, Università di Roma "Tor Vergata" \\ I.N.F.N., Sezione di Roma "Tor Vergata" \\ Via della Ricerca Scientifica, 100133 Roma ITALY
}

The perturbative analysis of models of open and closed superstrings presents a number of surprises. For instance, variable numbers of antisymmetric tensors ensure their consistency via generalized Green-Schwarz cancellations and a novel type of singularity occurs in their moduli spaces. All these features are related, in one way or another, to the presence of boundaries on the world sheet or, equivalently, of extended objects (branes) interacting with the bulk theory in space time. String dualities have largely widened the interest in these models, that exhibit a wealth of generic non-perturbative features of String Theory.

\section{INTRODUCTION}

The basic rules determining the perturbative spectra of oriented closed strings essentially amount to two basic constraints on their torus amplitudes, modular invariance and spinstatistics [1]. The former makes the choice of world-sheet "time" immaterial, while the latter fixes the relative contributions of Bose and Fermi fields. By now familiar for almost a decade, these rules determine the $G S O$ projections [2] for vacua in several space-time dimensions with different numbers of space-time supersymmetries, and have deepened considerably our understanding of String Theory. On the other hand, models of open and closed strings, the very ones that marked the birth of the field [3], have long been of interest only to a small community [4]. To some extent, this uneven fate is to be traced to the restrictions on their gauge groups [5] and to the relative simplicity and elegance of the heterotic string [6].

In 1987 I suggested that a link between openstring models and symmetric models of oriented closed strings, exhibited by the only two examples known at the time, the bosonic string and the $S O(32)$ type-I model [7], be taken as a general building principle for their spectra [8]. The resulting connection, a parameter-space analogue of

*Work supported in part by E.E.C. Grant CHRX-CT930340. the orbifold construction [9], relates the type-IIB model of oriented closed strings [10] to its "open descendant", the $S O(32)$ model of Green and Schwarz. Subsequent work exhibited the role of boundary conditions in lower-dimensional models 11] and led to new classes of open-string spectra in ten and six dimensions 12, with corresponding patterns of Chan-Paton symmetry breaking.

Six-dimensional models promptly displayed a rewarding surprise: their low-energy spectra include variable numbers of antisymmetric tensors 12] that take part in a generalized anomaly cancellation mechanism [13]. These marked differences with respect to perturbative vacua of the heterotic string were correctly traced to the "parent" type-IIB string. Moreover, an analysis of the low-energy supergravity revealed the existence of peculiar singularities in the moduli spaces of tensor multiplets, that result in infinite gauge coupling constants [13]. This perturbative phenomenon, and its non-perturbative counterpart expected to present itself in heterotic models [14], have aroused some interest during the past year. The singularities reflect the presence in the vacuum of string excitations with vanishing tension [15], indicating some sort of string generalization of the Nambu-Jona Lasinio-Goldstone phenomenon. More general types of compactifications, with non-trivial vacuum values for scalar fields in the internal space, have been advocated to provide a geometrical framework for these re- 
sults [16].

String dualities [17], and in particular the strong-weak coupling relation in ten dimensions between the type-I $S O(32)$ model and the $\operatorname{Spin}(32) / Z_{2}$ heterotic string [18], have turned open strings into a central ingredient of the emerging picture. Most notably, Polchinski's observation 19] that extended objects, $D$ branes, where open-string endpoints terminate, have a central role as carriers of Ramond-Ramond charges, has provided a conformal field theory setting for some of the soliton studies [20] that have played a central role in the development of the string duality picture. The recent surge of interest in open-string vacua, accompanied by the emergence of a new term, "orientifold", that stresses the geometrical significance of this peculiar orbifold construction, has led to the construction of many more six-dimensional models 21 24. Four-dimensional vacua with $N=1$ supersymmetry are comparatively less understood 25.26], but [26] contains the first instance of a chiral model, with three generations of chiral fermions. The Kähler manifold of the untwisted scalars in the unoriented closed sector, $S p(8) /(S U(4) \times U(1))$, is strongly suggestive of a twelve-dimensional interpretation in the spirit of 16 .

The relation between closed models and their open descendants, a general property of Conformal Field Theory and current algebra, associates one or more classes of "descendants" 27,28] to the $A D E$ series [29] of minimal and $S U(2)$ WZW models [30]. The resulting models, also of some potential relevance to Condensed Matter Physics 31, reveal the occurrence of another exotic phenomenon: the open sector may possess a larger symmetry than the bulk theory 28].

This article is based on seminars presented at CERN, at the XXVIII Institute d'Ete of the Ecole Normale Superieure in Paris and at the XXX Ahrenshoop Symposium in Buckow. Since much literature is now available on $D$ branes and their applications [32], I shall confine my attention to some of the "miracles" of open-string perturbation theory that have shaped my own understanding of the subject. In the next Section I review briefly the scope of the original proposal, while displaying, in a couple of simple instances, the resulting structure of open-string spectra. The following Section illustrates the nature of the generalized Green-Schwarz mechanism, while displaying some corresponding properties of sixdimensional supergravity. The final Section describes, in the open descendants of the $D_{5}$ model of 29, the extension of internal symmetry in the open sector via simple currents of non-integer dimension [28].

\section{OPEN DESCENDANTS, OR ORIEN- TIFOLDS}

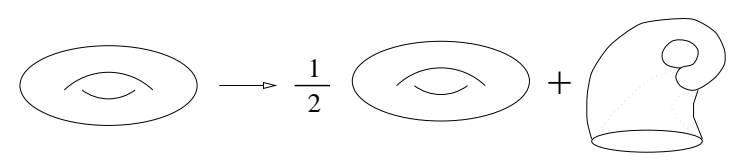

Figure 1. Projection of the closed spectrum

Let me begin by reviewing the original proposal of [8]. The basic idea (fig. 1) is to use worldsheet parity to project a consistent spectrum of oriented closed strings symmetric under the interchange of left and right modes. This operation mixes left and right modes, and its proper completion requires the Klein-bottle amplitude. As this violates modular invariance, singular (ultraviolet) contributions can result from the origin, the edge of moduli space, that in this case is a half-line. On the other hand, the Kleinbottle amplitude is "dual" to an amplitude describing the propagation of closed strings between two cross-caps, and the modular transformation relating these two pictures turns the ultraviolet divergences into infrared divergences related to massless exchanges. A suitable spectrum of open strings, the "twisted sector" of this construction, can dispose of the resulting singularities, thus restoring the consistency of the models. Indeed, Klein-bottle, annulus and Möbius strip, though vastly different, are all related by suitable modular transformations to "dual" amplitudes describing closed-string exchanges between pairs of boundaries and/or crosscaps. In particular (fig.

\footnotetext{
${ }^{2}$ In order to add a crosscap to a surface, one cuts from it a disk and glues to the resulting hole the single boundary of a Möbius strip.
} 
2 ), the annulus corresponds to a tube terminating at two boundaries, while the Möbius strip corresponds to a tube terminating at one boundary and one crosscap. As a result, the proper factorization of these amplitudes turns the consistency conditions into a set of linear equations (tadpole conditions) for the numbers of "colors", or types of Chan-Paton charges [5], associated to the various types of boundaries. Some of the tadpole conditions are related to unphysical exchanges and signal the presence of anomalies [33].

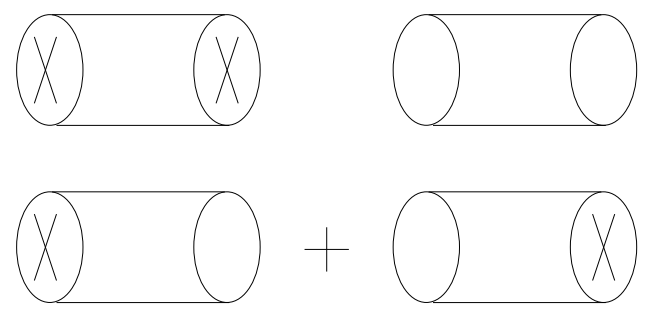

Figure 2. Klein bottle, annulus and Moebius strip

It is amusing to see how this pattern is realized, leading to whole new classes of conformal models. Let me begin with a few simple cases, and in particular with two classes of non-supersymmetric ten-dimensional models drawn from [12] and 34]. The models in the first class are described by

$$
\begin{aligned}
\mathcal{T} & =\left|O_{8}\right|^{2}+\left|V_{8}\right|^{2}+\left|S_{8}\right|^{2}+\left|C_{8}\right|^{2} \\
\mathcal{K} & =\frac{1}{2}\left(O_{8}+V_{8}-S_{8}-C_{8}\right) \\
\mathcal{A} & =\frac{n_{o}^{2}+n_{v}^{2}+n_{s}^{2}+n_{c}^{2}}{2} V_{8}+\left(n_{o} n_{v}+n_{s} n_{c}\right) O_{8} \\
& -\left(n_{v} n_{s}+n_{o} n_{c}\right) S_{8}-\left(n_{v} n_{c}+n_{o} n_{s}\right) C_{8} \\
\mathcal{M} & =-\frac{n_{o}+n_{v}+n_{s}+n_{c}}{2} \hat{V}_{8}
\end{aligned}
$$

where the characters of level-one $S O(8)$ modules are (orthogonal) combinations of fourth powers of Jacobi theta functions and of the Dedekind $\eta$ function

$$
\begin{array}{ll}
O_{8}=\frac{\theta_{3}^{4}+\theta_{4}^{4}}{2 \eta^{4}} & V_{8}=\frac{\theta_{3}^{4}-\theta_{4}^{4}}{2 \eta^{4}}, \\
S_{8}=\frac{\theta_{2}^{4}+\theta_{1}^{4}}{2 \eta^{4}} & C_{8}=\frac{\theta_{2}^{4}-\theta_{1}^{4}}{2 \eta^{4}},
\end{array}
$$

and where the "hatted" functions, of argument $-\sqrt{q}$, are redefined by phases and start with posi- tive coefficients [12,27]. In the unprojected closed sector, $\left|O_{8}\right|^{2}$ starts with a tachyon $T$ and $\left|V_{8}\right|^{2}$ starts with the usual collection of $g_{\mu \nu}, B_{\mu \nu}$ and $\phi$. In addition, there are two low-lying sectors of $R R$ bosons, $A^{+}{ }_{\mu \nu \rho \sigma}$, a four-form with self-dual field strength, a two-form $B^{\prime}{ }_{\mu \nu}$ and a scalar $\phi^{\prime}$ from $\left|S_{8}\right|^{2}$, and $A^{-}{ }_{\mu \nu \rho \sigma}$, a four-form with antiself-dual field strength, a two-form $B^{\prime \prime}{ }_{\mu \nu}$ and a scalar $\phi^{\prime \prime}$ from $\left|C_{8}\right|^{2}$. After the projection, one is left with $g, \phi$ and $T$ from the NS-NS sectors, and $B^{\prime}$ and $B^{\prime \prime}$ from the $R-R$ sectors. The open sector of eqs. (3) and (4) is chiral, since the left-handed fermions in $S_{8}$ and $C_{8}$ carry different types of quantum numbers. The tadpole conditions identify the dimensionalities of pairs of Chan-Paton charge spaces, setting $n_{o}=n_{v}$ and $n_{s}=n_{c}$. The model includes two two-forms, both involved in the anomaly cancellation mechanism, since the residual anomaly polynomial is

$$
\begin{aligned}
I_{A} & \sim\left(\operatorname{tr} F_{o}^{2}-\operatorname{tr} F_{v}^{2}\right)\left(\operatorname{tr} F_{s}^{4}-\operatorname{tr} F_{c}^{4}\right. \\
& \left.-\frac{1}{4} \operatorname{tr} R^{2}\left(\operatorname{tr} F_{s}^{2}-\operatorname{tr} F_{c}^{2}\right)\right) \\
& +\left(\operatorname{tr} F_{s}^{2}-\operatorname{tr} F_{c}^{2}\right)\left(\operatorname{tr} F_{o}^{4}-\operatorname{tr} F_{v}^{4}\right. \\
& \left.-\frac{1}{4} \operatorname{tr} R^{2}\left(\operatorname{tr} F_{o}^{2}-\operatorname{tr} F_{v}^{2}\right)\right) .
\end{aligned}
$$

Thus, $B^{\prime}$ and $B^{\prime \prime}$ share in a democratic fashion the crucial task of canceling the gauge anomalies, while there are no gravitational anomalies since there is no net number of chiral fermions.

A variant, corresponding to a different choice of Klein-bottle projection, was introduced in [34]. It is particularly interesting since it includes a $U(32)$ model that is not supersymmetric but is free of tachyons, an open analogue of the $S O(16) \times$ $S O(16)$ model of 35 . Indeed, the choice of Kleinbottle projection, determined by the "crosscap constraint" [27], is not unique in general, and the different choices correspond to vastly different spectra. In this case, taking

$\mathcal{K}=\frac{1}{2}\left(-O_{8}+V_{8}+S_{8}-C_{8}\right)$,

the projected closed spectrum contains $g$ and $\phi$ (but no tachyon) from the $N S-N S$ sectors, and $A^{+}, \phi^{\prime}$ and $B^{\prime \prime}$ from the $R-R$ sectors. 
The corresponding chiral open sector, described by

$\mathcal{A}=n \bar{n} V_{8}-\frac{n^{2}+\bar{n}^{2}}{2} C_{8}$

and

$\mathcal{M}=\frac{n+\bar{n}}{2} \hat{C}_{8}$

can be obtained permuting some of the charge assignments in eq. (3) and turning pairs of "real" charge spaces into pairs of "complex" spaces for the (anti)fundamental representations of a unitary group. The corresponding multiplicities are equal in pairs, and in this case $n=\bar{n}=32$. This recurrent possibility, related to the presence of simple currents in $^{\text {in }}$ the spectrum, was first noted in [12] and is studied in detail in [27].

The residual anomaly polynomial of this model

$$
\begin{aligned}
I_{A} & \sim\left(\operatorname{tr} F^{2}-\operatorname{tr} R^{2}\right)\left(\operatorname{tr} F^{4}+\frac{1}{8} \operatorname{tr} R^{4}\right. \\
& \left.+\frac{1}{32}\left(\operatorname{tr} R^{2}\right)^{2}-\frac{1}{8} \operatorname{tr} F^{2} \operatorname{tr} R^{2}\right) \\
& +\frac{2}{3}\left(\operatorname{tr} F^{3}-\frac{1}{8} \operatorname{tr} F \operatorname{tr} R^{2}\right)^{2} \\
& +\operatorname{tr} F\left(\frac{2}{5} \operatorname{tr} F^{5}+\frac{1}{240} \operatorname{tr} F \operatorname{tr} R^{4}\right. \\
& \left.-\frac{1}{192} \operatorname{tr} F\left(\operatorname{tr} R^{2}\right)^{2}\right)
\end{aligned}
$$

is particularly interesting. Together with the twoform $B^{\prime \prime}$, two more fields take part in a generalized Green-Schwarz mechanism, $A^{+}$and the eight-form dual to $\phi^{\prime}$. The coupling of the latter gives mass to the $U(1)$ vector field, and the actual low-energy gauge group is thus $S U(32)$. It should be noticed, in particular, that the selfduality of the field strength of $A^{+}$reflects itself in the nature of the corresponding term in eq. (10), a perfect square. We have thus come across the first miracle alluded to in the Introduction: the Green-Schwarz mechanism takes typically a more general form in these models, with contributions from forms of different degrees. This is actually the most complicated example I am aware of,

\footnotetext{
${ }^{3}$ Simple currents are operators with abelian fusion rules with all other fields in the theory. This notion was introduced in 36.
}

since the six-dimensional ones of 13 only include (anti)self-dual two-forms, and occasionally fourforms, in a way that extends the original fourdimensional mechanism of [37].

Let me now turn to a couple of additional instances. The first presents itself when one compactifies the type-I superstring to nine dimensions, and will serve the purpose of illustrating the role of $D$ branes in connection with $T$-duality. In this case

$\mathcal{T}=\left|V_{8}-S_{8}\right|^{2} \frac{\sum q^{\frac{\alpha^{\prime}}{4} p_{L}^{2}} \bar{q}^{\frac{\alpha^{\prime}}{4} p_{R}^{2}}}{\eta(q) \bar{\eta}(\bar{q})}$

where, as usual,

$p_{L, R}=\frac{m}{R} \pm \frac{n R}{\alpha^{\prime}}$,

describes the toroidal compactification of the Type-IIB superstring to nine dimensions. In the absence of Wilson lines, the familiar Klein-bottle projection

$\mathcal{K}=\frac{1}{2}\left(V_{8}-S_{8}\right) \frac{\sum q^{\alpha^{\prime} \frac{m^{2}}{2 R^{2}}}}{\eta\left(q^{2}\right)}$

leads to

$\mathcal{A}=\frac{N^{2}}{2}\left(V_{8}-S_{8}\right) \frac{\sum q^{\alpha^{\prime} \frac{m^{2}}{2 R^{2}}}}{\eta(\sqrt{q})}$

and

$\mathcal{M}=-\frac{N}{2}\left(\hat{V}_{8}-\hat{S}_{8}\right) \frac{\sum q^{\alpha^{\prime} \frac{m^{2}}{2 R^{2}}}}{\hat{\eta}(-\sqrt{q})}$,

where $N=32$ and, again, the power series of "hatted" functions starts with unit coefficient. The spectrum is clearly not invariant under $T$ duality, since $\mathcal{K}, \mathcal{A}$ and $\mathcal{M}$ are all drastically altered by the usual replacement of $R$ with $\alpha^{\prime} / R$. This should be contrasted with the familiar case of the heterotic string, where $T$ duality finds an elegant setting in the Narain lattice construction [38]. One can actually explain in rather simple terms what is happening [39]. Indeed, $T$ duality is an asymmetric parity operation on the world sheet, that transforms the (anti)holomorphic portions of the internal string coordinate according to $X_{L} \rightarrow X_{L}$ and $X_{R} \rightarrow-X_{R}$. Since the 
usual Neumann condition essentially associates the open-string sector to $X_{L}+X_{R}$, a $T$ duality turns it into a "Dirichlet" open sector corresponding to $X_{L}-X_{R}$.

Where do the "Dirichlet" ends terminate? We owe to 19] a pervasive geometrical characterization of these extended objects, now commonly referred to as $D$ branes. In this case, they are just hyperplanes, but a closer inspection reveals that they are actually dynamical objects, a variety of string solitons. There is already a vast literature on this important topic [32], and I shall thus confine my attention, as advertised, to their role in perturbative open-string spectra. Indeed, the first lesson is precisely that they are part of perturbative open-string spectra, so what better way do we have to gain some familiarity with their properties! The second observation is that, allowing for these objects, one is apparently going past the original restriction [8] to models with a leftright symmetric GSO projection. Actually, insofar as one is ready to perform suitable $T$-duality transformations, every situation is captured by the original setting. There is a simple, canonical example, that I find quite instructive. It is a generalization of the toroidal model of eqs. (14)-(15) where, following [40], one allows for Wilson lines breaking $S O(32)$ to $U(M) \times S O(32-2 M)$. The Wilson lines affect the energy levels via minimal couplings, so that

$$
\begin{aligned}
\mathcal{A} & =\left(\frac{N^{2}}{2}+M \bar{M}\right)\left(V_{8}-S_{8}\right) \frac{\sum q^{\alpha^{\prime} \frac{m^{2}}{2 R^{2}}}}{\eta(\sqrt{q})} \\
& +N M\left(V_{8}-S_{8}\right) \frac{\sum q^{\alpha^{\prime} \frac{(m+a R / 2)^{2}}{2 R^{2}}}}{\eta(\sqrt{q})} \\
& +N \bar{M}\left(V_{8}-S_{8}\right) \frac{\sum q^{\alpha^{\prime} \frac{(m-a R / 2)^{2}}{2 R^{2}}}}{\eta(\sqrt{q})} \\
& +\frac{M^{2}}{2}\left(V_{8}-S_{8}\right) \frac{\sum q^{\alpha^{\prime} \frac{(m+a R)^{2}}{2 R^{2}}}}{\eta(\sqrt{q})} \\
& +\frac{M^{2}}{2}\left(V_{8}-S_{8}\right) \frac{\sum q^{\alpha^{\prime}} \frac{(m-a R)^{2}}{2 R^{2}}}{\eta(\sqrt{q})} \\
\mathcal{M} & =-\frac{N}{2}\left(\hat{V}_{8}-\hat{S}_{8}\right) \frac{\sum q^{\alpha^{\prime} \frac{m^{2}}{2 R^{2}}}}{\hat{\eta}(-\sqrt{q})}
\end{aligned}
$$

$$
\begin{aligned}
& -\frac{M}{2}\left(\hat{V}_{8}-\hat{S}_{8}\right) \frac{\sum q^{\alpha^{\prime} \frac{(m+a R)^{2}}{2 R^{2}}}}{\hat{\eta}(-\sqrt{q})} \\
& -\frac{\bar{M}}{2}\left(\hat{V}_{8}-\hat{S}_{8}\right) \frac{\sum q^{\alpha^{\prime} \frac{(m-a R)^{2}}{2 R^{2}}}}{\hat{\eta}(-\sqrt{q})} .
\end{aligned}
$$

where $N+2 M=32$.

The Möbius contribution is only associated to terms of $\mathcal{A}$ with identical charges, but the corresponding momentum shifts are doubled, since in the proper parametrization its boundary has twice the length of each boundary of the annulus. All this is nicely consistent with the factorization of "dual" vacuum channels.

If one performs a $T$ duality transformation, the translations in momentum space turn into translations in coordinate space. As these are simple to visualize (fig. 3), the resulting $D$-brane picture, though equivalent, has undoubtedly some aesthetic appeal. For instance, it is evident from eqs. (16) and (17) that $a=\frac{1}{R}$ results in an enhancement (or rather, a partial recovery) of the gauge symmetry, that becomes $S O(32-2 M) \times S O(2 M)$, while $a=\frac{2}{R}$ retrieves the full $S O(32)$ gauge group. Referring to fig. 3 , in the $T$-dual picture the prototype breaking of eqs. (16) and (17) involves strings stretched between a $D$ brane $\left(D_{o}\right)$ at the bottom of the circle and an additional pair of $D$ branes at points symmetrically located with respect to the vertical $\left(D_{+}\right.$and $\left.D_{-}\right)$. The partial recovery of gauge symmetry takes place when the last two meet at the top of the circle, whereas full recovery is attained when all three meet at the bottom.

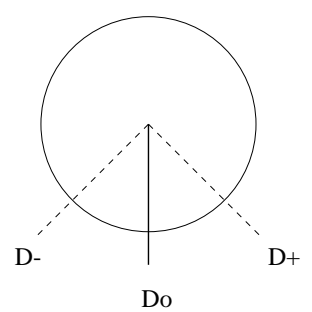

Figure 3. D-brane picture of Wilson-line breaking

One can actually modify the Klein-bottle projection, since the crosscap constraint [27] allows one to (anti)symmetrize internal momenta according to their quantum numbers. This interest- 
ing possibility, first exploited in 22] and discussed further in [24], corresponds to

$\mathcal{K}=\frac{1}{2}\left(V_{8}-S_{8}\right) \frac{\sum(-1)^{m} q^{\alpha^{\prime} \frac{m^{2}}{2 R^{2}}}}{\eta\left(q^{2}\right)}$

and leads to open descendants without an open sector. Indeed, familiar properties of theta functions imply that the transverse-channel amplitude corresponding to eq. (18) does not introduce any singularities, since it does not involve any massless exchange.

Let me now complicate matters slightly. Following 11], I shall describe how to associate open descendants to $c=1$ orbifolds. While the resulting conformal models can be associated to string compactifications, for simplicity I shall only consider the internal part. The novelty is that strings with Neumann and Dirichlet conditions coexist in the spectrum, independently of $T$ dualities. This geometrical setting has been discussed for the superstring in [21], with resulting constraints equivalent to those in [11]. The covariant form has the advantage of leading naturally to large Chan-Paton gauge groups. Indeed, in rational models one typically faces the presence of quantized backgrounds for the $N S-N S$ two-form, and these reduce the rank of the Chan-Paton group. For instance, starting from a total dimensionality 32 for the Chan-Paton charge space, a quantized two-form of (even) rank $r$ results in effective quantized Wilson lines that reduce the dimensionality of the charge space to $32 / 2^{r / 2} 40$.

The oriented closed sector [9] is built projecting the internal part of the torus amplitude (11) so as to enforce the identification between $X$ and $-X$ required by the geometry of the orbifold. The resulting "untwisted" sector breaks modular invariance, that is recovered associating a pair of "twisted" sectors to the two fixed points of the orbifold. The resulting torus amplitude is

$$
\begin{aligned}
& \mathcal{T}=\frac{1}{2} \frac{\sum q^{\frac{\alpha^{\prime}}{4} p_{L}^{2}} \bar{q}^{\frac{\alpha^{\prime}}{4} p_{R}^{2}}}{\eta \bar{\eta}}+\frac{1}{2}\left|\frac{\theta_{3} \theta_{4}}{\eta^{2}}\right| \\
& +\frac{1}{2}\left|\frac{\theta_{2} \theta_{3}}{\eta^{2}}\right|+\frac{1}{2}\left|\frac{\theta_{2} \theta_{4}}{\eta^{2}}\right| .
\end{aligned}
$$

In order to construct the unoriented closed sector of the descendant, one starts again from the
Klein bottle amplitude. In this case, however, an inspection of the sections allowed on the genusone surfaces [11] or, equivalently, of the operator content, reveals the need for both momentum and winding sums. As a result

$$
\begin{aligned}
\mathcal{K} & =\frac{1}{4} \frac{\sum_{m} q^{\alpha^{\prime} \frac{m^{2}}{2 R^{2}}}}{\eta\left(q^{2}\right)}+\frac{1}{4} \frac{\sum_{n} q^{\frac{n^{2} R^{2}}{2 \alpha^{\prime}}}}{\eta\left(q^{2}\right)} \\
& +\left(\frac{\theta_{2} \theta_{3}}{\eta^{2}}\left(q^{2}\right)\right)^{1 / 2}
\end{aligned}
$$

is invariant under $T$ duality transformations. The unitarity of the vacuum channels exposed by an $S$ transformation then requires that the open sector contain momentum and winding sums as well. Therefore, one has to face the simultaneous presence of $N N$ and $D D$ strings, since the former can have momentum, while the latter can have windings in the internal direction. It should be appreciated, however, that the endpoints of these $D D$ strings sit at the two fixed points of the orbifold. There are thus three types of $D D$ strings: with both ends at one or the other fixed point, or stretched between the two fixed points. This is a discrete version of the breaking of fig. 3, as pertains to the absence of corresponding moduli in the projected spectrum. Aside from the usual $N N$ strings (possibly with "quantized" Wilson lines), there are additional ones with one $N$ and one $D$ end, with the latter at any of the two fixed points. In conclusion,

$$
\begin{aligned}
\mathcal{A} & =\frac{\operatorname{tr}\left(\mathbf{1}_{N}\right)^{2}}{4} \frac{\sum_{m} q^{\alpha^{\prime} \frac{m^{2}}{2 R^{2}}}}{\eta} \\
& +\frac{\operatorname{tr}\left(\mathbf{R}_{N}\right)^{2}}{4}\left(\frac{\theta_{3} \theta_{4}}{\eta^{2}}\right)^{1 / 2} \\
& +\sum_{i=1,2} \frac{\operatorname{tr}\left(\mathbf{1}_{N}\right) \operatorname{tr}\left(\mathbf{1}_{D i}\right)}{4}\left(\frac{\theta_{2} \theta_{3}}{\eta^{2}}\right)^{1 / 2} \\
& +\sum_{i=1,2} \frac{\operatorname{tr}\left(\mathbf{R}_{N}\right) \operatorname{tr}\left(\mathbf{R}_{D i}\right)}{4}\left(\frac{\theta_{2} \theta_{4}}{\eta^{2}}\right)^{1 / 2} \\
& +\sum_{i, j=1,2} \frac{\operatorname{tr}\left(\mathbf{1}_{D i}\right)^{2}}{4} \frac{\sum_{n} q^{\alpha^{\prime}} \frac{(n+(i-j) / 2) R^{2}}{2 \alpha^{\prime}}}{\eta} \\
& +\sum_{i=1,2} \frac{\operatorname{tr}\left(\mathbf{R}_{D i}\right)^{2}}{4}\left(\frac{\theta_{3} \theta_{4}}{\eta^{2}}\right)^{1 / 2}
\end{aligned}
$$


and

$$
\begin{aligned}
\mathcal{M} & =\frac{\operatorname{tr}\left(\mathbf{1}_{N}\right)+\sum_{i=1,2} \operatorname{tr}\left(\mathbf{1}_{D i}\right)}{4}\left(\frac{\hat{\theta}_{3} \hat{\theta}_{4}}{\hat{\eta}^{2}}\right)^{1 / 2} \\
& +\sum_{i=1,2} \frac{\operatorname{tr}\left(\mathbf{1}_{D i}\right)}{4} \frac{\sum_{n} q^{\alpha^{\prime} \frac{(n+(i-j) / 2) R^{2}}{2 \alpha^{\prime}}}}{\hat{\eta}} \\
& +\frac{\operatorname{tr}\left(\mathbf{1}_{N}\right)}{4} \frac{\sum_{m} q^{\alpha^{\prime} \frac{m^{2}}{2 R^{2}}}}{\hat{\eta}}
\end{aligned}
$$

where $\operatorname{tr}(\mathbf{1})=Q_{+}+Q_{-}$and $\operatorname{tr}(\mathbf{R})=Q_{+}-Q_{-}$ implement a $Z_{2}$ breaking in the charge sectors. For brevity, I have omitted the arguments of all $\theta$ and $\eta$ functions, equal to $\sqrt{q}$ in the annulus amplitude and to $-\sqrt{q}$ in the Möbius amplitude.

It can be verified that the vacuum channels of $\mathcal{K}$ and $\mathcal{A}$ comprise contributions of the different sectors with squared reflection coefficients, and that, as usual [12], the coefficients in vacuum channel of $\mathcal{M}$ are geometric means of them. All this, of course, if the $D$ charges sit at the fixed points, and if a corresponding quantization holds for the Wilson lines acting on $N$ charges, that I have not displayed in eq. (22). In space-time models, tadpole conditions related to untwisted massless exchanges constrain the dimensionality of the charge space, whereas others related to twisted exchanges constrain the breaking pattern. Actually, the choice in eqs. (21) and (22) is not unique, and an additional one may be obtained interchanging $\operatorname{Tr}(\mathbf{1})$ and $-\operatorname{Tr}(\mathbf{R})$, while identifying pairs of Chan-Paton charge spaces. This is one more instance of the phenomenon discussed at length in 12] and [27] and already encountered in eq. (8), whereby pairs of "real" charge spaces associated to orthogonal or symplectic groups are traded for pairs of conjugate spaces of "complex" charges associated to unitary groups.

In the open sector $T$ duality is not necessarily a symmetry, since it involves the interchange of $N$ and $D$ charges, but it can be if identical breaking patterns are chosen for the $N$ and $D$ charge spaces. In the former, the breaking is controlled by (quantized) Wilson lines, while in the latter it is determined by the distribution of $D$ charges among the two fixed points. This has been discussed at length in 21 233. In modern parlance, this model exhibits the coexistence, again in the perturbative spectrum, of $D$ branes of different dimensions. Part of this structure was anticipated long ago in the low-energy analysis of [41], that motivated the string analysis of 11].

\section{TENSOR MULTIPLETS IN SIX DI- MENSIONS}

Let me now turn to six dimensions, and in particular to type-I models with simple supersymmetry. They are descendants of the unique $K 3$ reduction of the Type-IIB string [42], whose lowenergy dynamics is described by $N=2 b$ supergravity [43] with $21 N=2 b$ tensor multiplets and a moduli space $S O(5,21) / S O(5) \times S O(21)$. There are only two relevant $N=2 b$ multiplets: the gravitational multiplet includes two left-handed Weyl gravitini, the gravitational field and five self-dual two-tensors, while the tensor multiplet includes a single antiself-dual tensor, two right-handed Weyl spinors and five scalar fields. The $N=2 b$ supergravity was constructed, to lowest order in Fermi fields, in [43], while the unique anomaly-free spectrum was identified in 44.

In proceeding to the open descendants, the novelty is that the projection can result in models with simple supersymmetry in six dimensions with different numbers of residual two-tensors. In simple superymmetry, the gravitational multiplet includes the gravity field, a left-handed Weyl gravitino and a self-dual two-form, while the tensor multiplet includes an antiself-dual two-form, a right-handed Weyl spinor and a scalar. In this article we shall also consider the vector multiplet, that includes a vector and a left-handed Weyl spinor.

There is a particularly simple class of models, originally constructed in [12], where I first came across the generalized Green-Schwarz mechanism. Many similar models have been recently discussed in 22 24. In particular, 24 discusses the only string vacuum I am aware of with no tensor multiplets at all. Our simple example is built starting from the toroidal reduction of the Type-IIB

\footnotetext{
${ }^{4}$ In this article I am abiding to present-day notation, whereas in 43 and 13] simple supersymmetry was referred to as $N=2 b$.
} 
string on the $S O(8)$ lattice. In the notation of the previous Section, the torus amplitude reads

$\mathcal{T}=\left|V_{8}-S_{8}\right|^{2}\left(\left|O_{8}\right|^{2}+\left|V_{8}\right|^{2}+\left|S_{8}\right|^{2}+\left|C_{8}\right|^{2}\right)$.

The next step is a conventional $Z_{2}$ orbifold [9], where one reverses simultaneously the sign of the four internal fermions $\psi^{i}$ and of four of the eight internal fermions that bosonize the internal coordinates. Precisely in the spirit of the fermionic construction in [1], this is manifestly compatible with the corresponding fermionic supercurrent. The characters break accordingly into pairs with opposite eigenvalues under the $Z_{2}$ projection. Thus, for instance,

$V_{8}-S_{8} \rightarrow\left(V_{4} 0_{4}-C_{4} C_{4}\right)+\left(0_{4} V_{4}-S_{4} S_{4}\right)$,

where the two terms, denoted $Q_{o}$ and $Q_{v}$ in [12 have, respectively, positive and negative eigenvalues under the involution, and the resulting torus amplitude for the Type-IIB string on the orbifold,

$\mathcal{T}=\sum_{i=1}^{16}\left|\chi_{i}\right|^{2}$

is a diagonal combination of 16 characters. The identity is

$\chi_{1}=Q_{o} O_{4} O_{4}+Q_{v} V_{4} V_{4}$,

and the remaining characters are simply determined after a modular $S$ transformation of this expression. Building the "standard" descendant is also simple, since the "parent" model is diagonal. Indeed, in this case Cardy 45] has related the spectrum of boundary operators to the fusionrule coefficients, an observation turned in 12] into a set of rules for building descendants of rational models. Thus, the open sector corresponding to (25) includes 16 charge sectors and

$\mathcal{A}=\frac{1}{2} \sum_{i j k} n^{i} n^{j} N_{i j}^{k} \chi_{k}$,

where $N_{i j}^{k}$ are the fusion-rule coefficients. Taking into account the five tadpole conditions, one is left with a number of models, the simplest of which has gauge group $S p(8)^{\otimes 4}$.

The closed spectrum of this class of models includes five tensor multiplets, and a direct analysis of the anomaly polynomial led to a big surprise. Its irreducible part cancels as usual after imposing the tadpole conditions, but one is left with the residual contribution

$I_{A} \sim \sum_{x, y} \eta_{r s} c_{x}^{r} c_{y}^{s} \operatorname{Tr}_{x} F^{2} \operatorname{Tr}_{y} F^{2}$,

where the labels refer to the simple factors of the gauge group, that does not factorize. Here $\eta$ is a Minkowski metric of signature $\left(1-n_{T}\right)$, with $n_{T}$ the number of tensor multiplets, and the $c^{\prime} s$ are constants. I have not indicated explicitly the gravitational contribution, but this could be done extending the range of $(x, y)$, at the price of including corresponding higher-derivative terms. This setting is thus more general than the original one considered by Green and Schwarz [7], since it demands Chern-Simons couplings for several antisymmetric tensors. Moreover, since the anomaly polynomial is quadratic, for the gauge part the mechanism is realized at the level of two-derivative terms. The analysis of the corresponding low-energy supergravity is thus bound to present some peculiarities, and indeed it does. In the first of 13 I constructed its field equations to lowest order in the Fermi fields requiring that the resulting effective action be invariant under supersymmetry. On the other hand, in the second paper of [13] the field equations are derived from the Wess-Zumino consistency conditions [46, extending previous studies of anomalous models in global supersymmetry 477. The choice affects the gauge current, whose divergence gives in the former case the "covariant" anomaly and in the latter the more satisfactory "consistent" anomaly. The current is not conserved, since in this model fermion loops are to cancel the residual, factorized anomaly. Of course, this is just Noether's theorem at work, and similar considerations apply to the Green-Schwarz setting of [7], where this inconsistency is present in higher-derivative couplings.

For each choice for the gauge current $J$, the vector field equation takes the form

$v_{r} c_{z}^{r} D_{\mu} F_{z}{ }^{\mu \nu}=J_{z}{ }^{\nu}$

for each factor $z$ of the gauge group, where $v_{r}$ are (redundant) coordinates for the scalar fields of the 
tensor multiplets, that live on the hyperboloid

$\eta^{r s} v_{r} v_{s}=1$.

Since this describes the coset $S O\left(1, n_{T}\right) / S O\left(n_{T}\right)$, the $S O\left(1, n_{T}\right)$ symmetry is spontaneously broken in the vacuum to $S O\left(n_{T}\right)$. Even the latter symmetry, however, is affected by the couplings $c_{z}^{r}$ induced by the anomaly, and as result the effective gauge couplings

$\frac{1}{g_{(z)}^{2}}=v_{r} c_{z}^{r}$

in the vector kinetic term corresponding to (29),

$L=-\frac{e}{2} v_{r} c_{z}^{r} \operatorname{tr}_{z}\left(F_{\mu \nu} F^{\mu \nu}\right)$

can blow up at some points of moduli space 13. This unusual feature, exploited in [14] in the context of six-dimensional heterotic-heterotic dualities, was ascribed in [15] to a novel phenomenon: the singularities signal the presence, in the vacuum, of a new type of excitation, a tensionless string. The argument is based on the structure of six-dimensional central charges and on the second-order tensor equation of [13], whose source term is the instanton density. In this analogue of the familiar Nambu-Jona Lasinio-Goldstone transition, where a particle becomes massless, one expects a whole tower of higher-spin excitations to play a role. This is the second of the peculiarities alluded to in the Introduction.

Let me stress once more that the vector couplings to the scalars of tensor multiplets induced by the anomaly are revealed by a perturbative analysis of type-I vacua, where the dilaton sits in a hypermultiplet. On the other hand, they are quite surprising if referred to the heterotic string, where the dilaton sits in a tensor multiplet, since the vector kinetic terms corresponding to eq. 29) contain non-perturbative contributions [14, 15. For instance, if a single tensor multiplet is present one can solve the constraint of eq. (30) in terms of hyperbolic functions of a scalar. In the heterotic case, this is to be identified with the dilaton, and the vector kinetic term corresponding to eq. (29)

$L_{V}=-\frac{e}{2}\left(c_{0} e^{-\phi}+c_{1} e^{\phi}\right) \operatorname{tr} F_{\mu \nu} F^{\mu \nu}$ includes the non-perturbative coupling described by $c_{1}$.

A simple formula, first derived in [26], follows from the ten-dimensional duality conjecture of 18] and relates the dilatons of heterotic and Type-I vacua:

$\phi_{I}^{(d)}=\frac{6-d}{4} \phi_{H}^{(d)}-\frac{d-2}{16} \log \operatorname{det} G_{H}^{(10-d)}$

It should be noted that the two dilatons are independent precisely in six dimensions. Though quite surprising from the heterotic viewpoint, it is thus natural to construct Type-I vacua without tensor multiplets 24]. More amusingly, one is naturally led to speculate that, in their duals, the "missing" dilaton has been effectively stabilized by non-perturbative effects. This point is worthy of a closer look, since it would realize in a simple setting a phenomenon long advocated for four-dimensional string physics.

\section{THE SYMMETRY OF BOUNDARY STATES}

I shall conclude with a brief discussion of the last exotic phenomenon mentioned in the Introduction, while confining my attention to the rational descendants of a rational conformal theory. In principle, one would expect that the open sector, determined by reflections at the ends of the tube, inherit at most the diagonal part of the symmetry algebra (Virasoro or extended) $A \times \bar{A}$ of the bulk theory. Rather surprisingly, however, in some instances the symmetry of boundary states turns out to be larger. This phenomenon was first noted [28] in the open descendants of the $D_{5}$ model of the $A D E$ classification of [29].

It is convenient to assume that the bulk spectrum has been resolved into independent components, so that the torus partition function is a permutation invariant built out of $m$ characters,

$\mathcal{T}=\sum_{i=1}^{m} \bar{\chi}_{i} \chi_{\sigma(i)}$

The relevant techniques have been developed in [48]. In the diagonal case the canonical choice

${ }^{5}$ Diagonal means, effectively, that $\mathcal{T}$ is built from the charge-conjugation modular invariant, i.e. $\sigma(j)=c(j)$. 
for the annulus of eq. 27 [45, 12 reflects the one-to-one correspondence between chiral sectors of the bulk spectrum and boundary states. It is also nicely consistent with factorization [12], since the Verlinde formula guarantees that in the corresponding vacuum channel $\tilde{\mathcal{A}}$, exposed by an $S$ modular transformation, each sector enters with a coefficient that is a perfect square:

$\tilde{\mathcal{A}} \sim \sum_{i} \chi_{i}\left(\sum_{j} \frac{S_{i j} n^{j}}{\sqrt{S_{1 i}}}\right)^{2}$.

In Conformal Field Theory, the annulus amplitude may be regarded as a generating function for the multiplicities of boundary operators that mediate between boundary states. Thus, in the diagonal case each chiral sector has $m$ independent reflection coefficients for the $m$ choices of boundary states at the ends of the tube, all expressible in terms of the $S$ matrix.

The $A_{7}$ model of 29]

$$
\begin{aligned}
\mathcal{T}_{A_{7}} & =\left|\chi_{1}\right|^{2}+\left|\chi_{2}\right|^{2}+\left|\chi_{3}\right|^{2}+\left|\chi_{4}\right|^{2}+\left|\chi_{5}\right|^{2} \\
& +\left|\chi_{6}\right|^{2}+\left|\chi_{7}\right|^{2}
\end{aligned}
$$

where the labels of the seven level-six characters are $2 I+1$, with $I$ their isospins, is a convenient example with unextended symmetry. On the other hand, the $D_{6}$ model of [29] has level eight and

$\mathcal{T}_{D_{6}}=\left|\chi_{1}+\chi_{9}\right|^{2}+\left|\chi_{3}+\chi_{7}\right|^{2}+2\left|\chi_{5}\right|^{2}$,

is a simple model with extended symmetry. In this case, there is a fixed-point ambiguity, resolved assigning two different fields to the term with multiplicity 2 . Together with the other combinations of level-eight $S U(2)$ characters in eq. (38), these define a fusion algebra with some $N_{i j}^{k}$ larger than one. As a result, some terms occur in $\mathcal{A}_{D_{6}}$ with multiplicities. This result reflects the existence of inequivalent three-point functions. The simplest instance of this phenomenon is quite familiar: in $S U(3)$ there are two invariant tensors with three adjoint indices, the structure constants $f^{a b c}$ and the symmetric tensor $d^{a b c}$.

In open descendants of off-diagonal models, the direct correspondence between boundary states and chiral sectors of the bulk spectrum is lost, since in this case the tube can only support a portion of them. In particular, if the boundaries are to preserve the bulk symmetry, the allowed sectors are those paired with their conjugates in the torus modular invariant. Thus, some of the reflection coefficients vanish, a condition naturally met by suitable linear combinations of the "diagonal" boundary states. However, not all boundary states are obtained in this way. For instance, in all $D_{\text {odd }}$ models one has a simple current of half-integer dimension, and the proper boundary states are combinations of pairs of "diagonal" ones invariant under the action of this simple current [28]. This is quite reminiscent of the structure of the first two terms in eq. (38), and is effectively an extension by the simple current, that in these models has non-integer dimension! As in the more familiar bulk case, the boundary state naively invariant under the action of the simple current actually splits into a pair of states. For instance, in the $D_{5}$ case the torus modular invariant is built out of the level-six characters of eq. (37), but

$$
\begin{aligned}
\mathcal{T}_{D 5} & =\left|\chi_{1}\right|^{2}+\left|\chi_{3}\right|^{2}+\left|\chi_{5}\right|^{2}+\left|\chi_{7}\right|^{2} \\
& +\left|\chi_{4}\right|^{2}+\chi_{2} \bar{\chi}_{6}+\chi_{6} \bar{\chi}_{2},
\end{aligned}
$$

and therefore one would expect a total of five boundary states. However, there are only three combinations of "diagonal" boundary states invariant under the action of the simple current, $(1+7),(2+6)$ and $(3+5)$. The correct total number, five, is thus recovered precisely because the state corresponding to the middle field splits into a pair $4_{+}$and $4_{-}$. The construction in 27 recovers quite naturally the pair of split fields, but there is also an analogue of the multiple threepoint functions of conventional bulk extensions. This is directly induced by the fusion of pairs of "off-diagonal" boundary states, and has a direct consequence for the annulus amplitude, that contains multiplicities. For instance, the conventional (real) choice for the $D_{5}$ annulus amplitude

$$
\begin{aligned}
& 2 A_{D_{5}}=\chi_{1}\left(n_{1}^{2}+n_{2}^{2}+n_{3}^{2}+n_{4_{+}}^{2}+n_{4_{-}}^{2}\right)+ \\
& \left(\chi_{2}+\chi_{6}\right)\left(2\left(n_{1} n_{2}+n_{2} n_{3}+n_{4_{+}} n_{3}+n_{4_{-}} n_{3}\right)\right)+ \\
& \chi_{3}\left(n_{2}^{2}+2 n_{3}^{2}+2\left(n_{2} n_{4_{+}}+n_{2} n_{4_{-}}+\right.\right. \\
& \left.\left.n_{4_{+}} n_{4_{-}}+n_{1} n_{3}\right)\right)+\chi_{4}\left(4 n_{2} n_{3}+2\left(n_{1} n_{4_{+}}+\right.\right.
\end{aligned}
$$




$$
\begin{aligned}
& \left.\left.n_{4_{+}} n_{3}+n_{1} n_{4_{-}}+n_{4_{-}} n_{3}\right)\right)+\chi_{5}\left(n_{2}^{2}+n_{4_{+}}^{2}+\right. \\
& \left.n_{4_{-}}^{2}+2 n_{3}^{2}+2\left(n_{2} n_{4_{+}}+n_{2} n_{4_{-}}+n_{1} n_{3}\right)\right)+ \\
& \chi_{7}\left(n_{1}^{2}+n_{2}^{2}+n_{3}^{2}+2 n_{4_{+}} n_{4_{-}}\right)
\end{aligned}
$$

contains multiplicities in $\chi_{3}$ and $\chi_{5}$, and the whole $D_{\text {odd }}$ series follows a similar pattern.

I would like to thank the Organizers of the XXX Ahrenshoop Meeting for their kind invitation to present this material. I am very grateful to Massimo Bianchi, Gianfranco Pradisi and Yassen S. Stanev for a long and enjoyable collaboration. I am also grateful to Carlo Angelantonj, Sergio Ferrara, Ruben Minasian and Fabio Riccioni for extensive discussions and an enjoyable collaboration over the past year.

\section{REFERENCES}

1. I. Antoniadis, C.Bachas and C. Kounnas, Nucl. Phys. B288 (1987) 87; I. Antoniadis and C. Bachas, Nucl. Phys. B298 (1988) 586; H. Kawai, D.C. Lewellen and S.-H. Tye, Nucl. Phys. B288 (1987) 1; W. Lerche, D. Lüst and A.N. Schellekens, Nucl. Phys. B287 (1987) 477; K.S. Narain, M.H. Sarmadi and C. Vafa, Nucl. Phys. B288 (1987) 551.

2. F. Gliozzi, J. Scherk and D. Olive, Nucl. Phys. B122 (1977) 253.

3. G. Veneziano, Nuovo Cimento 57A (1968) 190.

4. A (partial) list of references is:

V. Alessandrini, Nuovo Cimento 2A (1971) 321; V. Alessandrini and D. Amati, Nuovo Cimento 4A (1971) 222; E. Cremmer and J. Scherk, Nucl. Phys. B50 (1972) 509; L. Clavelli and J. Shapiro, Nucl. Phys. B57 (1973) 490; A. Chodos and C. Thorn, Nucl. Phys. B54 (1974) 509; E. Corrigan and D. Fairlie, Nucl. Phys. B91 (1975) 545; W. Siegel, Nucl. Phys. B109 (1976) 244; G. Venturi, Nuovo Cimento 16A (1976) 484; H. Dorn and H.J. Otto, Z. Phys. C32 (1986) 599; M.R. Douglas and B. Grinstein, Phys. Lett. B183 (1987) 52; S. Weinberg, Phys. Lett. B183 (1987) 287; J.A. Harvey and J.A. Minahan, Phys. Lett. B188 (1987) 44; C.G. Callan, C. Lovelace, C. Nappi and S.A. Yost, Nucl. Phys.
B308 (1988) 221; J. Polchinski and Y. Cai, Nucl. Phys. B296 (1988) 91; N. Ishibashi and T. Onogi, Nucl. Phys. B318 (1989) 239; P. Horava, Nucl. Phys. B327 (1989) 461, Phys. Lett. B231 (1989) 251; N. Ishibashi, Mod. Phys. Lett. A4 (1989) 251; Z. Bern and D.C. Dumbar, Nucl. Phys. B319 (1989) 104; J. Dai, R.G. Leigh and J. Polchinski, Mod. Phys. Lett. A4 (1989) 2073; J. Gowaerts, Phys. Lett. B220 (1989) 77, Int. J. Mod. Phys. A4 (1990) 4353;

additional references may be found in:

M.B. Green, J.H. Schwarz and E. Witten, Superstring Theory, Cambridge Univ. Press, Cambridge, 1987).

5. J.E. Paton and H.M. Chan, Nucl. Phys. B10 (1969) 516; J.H. Schwarz, in Current Problems in Particle Theory, Proc. J. Hopkins Conf. 6, Florence, 1982; N. Marcus and A. Sagnotti, Phys. Lett. B119 (1982) 97, B188 (1987) 58.

6. D.J. Gross, J.A. Harvey, E. Martinec and R. Rohm, Nucl. Phys. B256 (1985) 253.

7. M.B. Green and J.H. Schwarz, Phys. Lett. B149 (1984) 117.

8. A. Sagnotti, in Non-Perturbative Quantum Field Theory, eds. G. Mack et al, Pergamon Press, New York, 1988, p. 521.

9. L. Dixon, J.A. Harvey, C. Vafa and E. Witten, Nucl. Phys. B261 (1985) 678, B274 (1986) 285.

10. J.H. Schwarz, Nucl. Phys. B226 (1983) 289; P.S. Howe and P.C. West, Nucl. Phys. B238 (1984) 181.

11. G. Pradisi and A. Sagnotti, Phys. Lett. B216 (1989) 59.

12. M. Bianchi and A. Sagnotti, Phys. Lett. B247 (1990) 517, Nucl. Phys. B361 (1991) 519.

13. A. Sagnotti, Phys. Lett. B294 (1992) 196; S. Ferrara, R. Minasian and A. Sagnotti, Nucl. Phys. B474 (1996) 323.

14. M.J. Duff, R. Minasian and E. Witten, hepth/9601036.

15. N. Seiberg and E. Witten, hep-th/9603003; M.J. Duff, H. Lü and C. Pope, hepth/9603037.

16. C. Vafa, hep-th/9602022; D. Morrison and C. Vafa, hep-th/96020114, 9603161. 
17. Several reviews are now available:

M.J. Duff, hep-th/9608117; J.H. Schwarz, hep-th/9607201; A. Sen, hep-th/9609176; P.K. Townsend, hep-th/9612121.

18. E. Witten, Nucl. Phys. B443 (1995) 85.

19. J. Polchinski, Phys. Rev. Lett. 75 (1995) 4724.

20. M.J. Duff, R. Khuri and J. Lu, Phys. Reports 259 (1995) 213.

21. E. Gimon and J. Polchinski, hep-th/9601038; M. Berkooz, R. Leigh, J. Polchinski, J.H. Schwarz, N. Seiberg and E. Witten, hepth/9605184.

22. A. Dabholkar and J. Park, hep-th/9602030, 9604178, 9607041; E. Gimon and C.V. Johnson, hep-th/9604129, 9606176.

23. J. Blum and A. Zaffaroni, hep-th/9607019; J. Polchinski, hep-th/9606165; A. Sen, hepth/9605150; K. Dasgupta and S. Mukhi, hepth/9606044.

24. C. Angelantonj, M. Bianchi, G. Pradisi, A. Sagnotti and Ya.S. Stanev, Phys. Lett. B387 (1996) 743.

25. M. Berkooz and R.G. Leigh, hep-th/9605049.

26. C. Angelantonj, M. Bianchi, G. Pradisi, A. Sagnotti and Ya.S. Stanev, Phys. Lett. B385 (1996) 96; M. Bianchi, S. Ferrara, G. Pradisi, A. Sagnotti and Ya.S. Stanev, Phys. Lett. B387 (1996) 64.

27. M. Bianchi, G. Pradisi and A. Sagnotti, Phys. Lett. B273 (1991) 389; D. Fioravanti, G. Pradisi and A. Sagnotti, Phys. Lett. B321 (1994) 349; G. Pradisi, A. Sagnotti and Ya.S. Stanev, Phys. Lett. B354 (1995) 279, B356 (1995) 230.

28. G. Pradisi, A. Sagnotti and Ya.S. Stanev, Phys. Lett. B381 (1996) 97; G. Pradisi, hepth/9603104; A. Sagnotti and Ya.S. Stanev, hep-th/9605042.

29. A. Cappelli, C. Itzykson and J.B. Zuber, Comm. Math. Phys. 113 (1987) 1.

30. E. Witten, Comm. Math. Phys. 92 (1984) 455; V.G. Knizhnik and A.B. Zamolodchikov, Nucl. Phys. B247 (1984) 83.

31. A.W.W. Ludwig, Int. J. Mod. Phy. B8 (1994) 347; I. Affleck, cond-matt/9512099.

32. J. Polchinski, S. Chauduri and C.V. Johnson, hep-th 9602052;J. Polchinski, hepth/9611050; C. Bachas, hep-th/9701019.

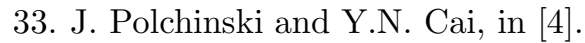

34. A. Sagnotti, in SUSY '95, eds. I. Antoniadis and H. Videau, Editions Frontieres, Paris, 1996, p. 473, hep-th/9509080.

35. L. Alvarez-Gaumé, P.Ginsparg, G. Moore and C. Vafa, Phys. Lett. B171 (1986) 155.

36. A.N. Schellekens and S. Yankielowicz, Nucl. Phys. B327 (1989) 673.

37. E. Witten, Phys. Lett. B149 (1984) 351; M. Dine, N. Seiberg and E. Witten, Nucl. Phys. B289 (1987) 589.

38. K.S. Narain, Phys. Lett. B169 (1986) 41; K.S. Narain, M.H. Sarmadi and E. Witten, Nucl. Phys. B279 (1987) 369.

39. J. Dai, R.G. Leigh and J. Polchinski, in 田; M.B. Green in [4]; P. Horava in [4].

40. M. Bianchi, G. Pradisi and A. Sagnotti, Nucl. Phys. B376 (1992) 365.

41. J.A. Harvey and J.A. Minahan, in 四.

42. For a review on K3, see: P.S. Aspinwall, hep-th/9611137.

43. L.J. Romans, Nucl. Phys. B276 (1986) 71.

44. L. Alvarez-Gaumé and E. Witten, Nucl. Phys. B234 (1983) 269.

45. J.L. Cardy, Nucl. Phys. B324 (1989) 581.

46. J. Wess and B. Zumino, Phys. Lett. B37 (1971) 95.

47. G. Girardi, R. Grimm and R. Stora, Phys. Lett. B156 (1985) 203; L. Bonora, P. Pasti and M. Tonin, Phys. Lett. B156 (1985) 341; E. Guadagnini, K. Konishi and M. Mintchev, Phys. Lett. B157 (1985) 37; N.K. Nielsen, Nucl. Phys. B244 (1984) 499; H. Itoyama, V.P. Nair and H. Ren, Nucl. Phys. B262 (1985) 317; E. Guadagnini and M. Mintchev, Nucl. Phys. B269 (1986) 543; C.L. Bilchak, R. Gastmans and A. van Proyen, Nucl. Phys. B273 (1986) 46; S. Ferrara, A. Masiero, M. Porrati and R. Stora, Nucl. Phys. B417 (1994) 238.

48. J. Fuchs, A.N. Schellekens and C. Schweigert, Nucl. Phys. B473 (1996) 323; hep-th/9612093. 